\title{
Matemática, Computação e Braille: Desafios da Pedagogia, da Semiótica e da Síntese da Fala
}

\author{
Angelica F. S. Dias ${ }^{1,2}$, Juliana B. S. França ${ }^{1}$, José Antonio S. Borges ${ }^{2,3}$, Júlio Tadeu \\ C. Silveira ${ }^{2}$, Marcos Fialho de Carvalho ${ }^{2,3}$, Marcos R. S. Borges ${ }^{1}$ \\ ${ }^{1}$ Programa de Pós-graduação em Informática/PPGI(UFRJ) \\ ${ }^{2}$ Instituto Tércio Pacitti da Universidade Federal do Rio de Janeiro (UFRJ) \\ ${ }^{3}$ HCTE - História das Ciências e das Técnicas e Epistemologia (UFRJ) \\ \{angelica, juliana.frança, mborges\}@ppgi.ufrj.br, \{julio,fialho,antonio2\}@nce.ufrj.br
}

\begin{abstract}
The construction of computerized systems has allowed the inclusion of students with visual impairment in high school and higher education classrooms. However, speech synthesizers were not ready to read math texts. This work goal is to show the solutions and methodology, based on the adoption of AsciiMath coding, a fast alternative to the use of LaTeX and the provision of a small compiler that takes mathematical expressions and creates sentences that are sonically unambiguous. This study enables, with appropriate tools, a blind student to use the computer to write and read math in an efficient and friendly way.
\end{abstract}

Resumo. A construção de sistemas computadorizados possibilitou a inserção de alunos com deficiência visual no ensino médio e superior nas salas de aula. No entanto, os sintetizadores de voz ainda não são preparados para ler textos de matemática. O objetivo deste trabalho é descrever estratégias baseadas na adoção da codificação AsciiMath, alternativa rápida ao uso do LaTeX e de disponibilização de um pequeno compilador que toma expressões matemáticas e cria frases que são sonoramente não ambíguas. Este estudo, através de ferramentas adequadas, permite que um aluno cego use o computador para escrever e ler matemática de forma eficiente.

\section{Introdução}

Nos dias de hoje diversas áreas do ensino para pessoas com deficiência visual no Brasil utilizam editores de texto computadorizados com síntese de voz para atender a alunos com deficiência visual. Tais sintetizadores vêm pré-configurados para produzir uma tradução razoavelmente precisa, tanto em termos fonéticos quanto prosódicos, de textos convencionais na língua portuguesa, permitindo uma leitura fluente (Ferreira, 2014). Isso não ocorre no ensino de matemática para cegos, pois a escrita matemática segue regras muito peculiares quando é falada, tanto por ser tipicamente não linear, quanto por exibir ambiguidades que exigem um tratamento prosódico especial. Por exemplo, $2^{1 / 2}$ é falado diferentemente de $21 / 2$. (ECS, 2015).

Este texto descreve as iniciativas computacionais realizadas com alunos de graduação de engenharia da Computação de uma universidade federal, juntamente com alunos de ensino médio, no sentido de tornar viável, acessível e compartilhável a escrita e leitura de textos avançados de matemática no computador que foram consolidadas no 
VII Congresso Brasileiro de Informática na Educação (CBIE 2018)

Anais do XXIX Simpósio Brasileiro de Informática na Educação (SBIE 2018)

sistema Dosvox e outras ferramentas específicas como o sistema Intermat. Apresenta também a integração com algumas soluções para transcrição para Braille Matemático Unificado (padrão usado no Brasil e Portugal) e algumas questões relacionadas à educação inclusiva e aprendizagem colaborativa mediada por computador, entre alunos com ou sem deficiência visual. São mostradas algumas dificuldades encontradas e sugestões para trabalhos futuros.

\section{Ensino de Matemática para Alunos Deficientes Visuais}

Para a matemática dos primeiros anos do ensino fundamental, existe suporte no Sistema Dosvox (Borges, 2009; Dias et al 2016) e no Braille Fácil (Borges e Junior, 2001). Estas soluções vêm sendo efetivas no auxílio aos processos de ensino e aprendizagem. (Pereira e Rodrigues, 2013). Na verdade, este mesmo problema ocorre também no ensino para alunos não cegos, onde ferramentas especializadas (LaTeX) são adotadas na escrita e leitura não linear. Assim, o ensino da matemática vem utilizando como suporte a escrita Braille, que embora seja intrinsecamente unidimensional, é capaz de prover mecanismos de manipulação de textos não lineares. Mas há uma grande dificuldade: quase nunca os professores das classes convencionais estão habilitados para esta forma de transcrição.

É preciso também levar em conta que o Braille matemático não é universal, sendo que o Brasil adotou um código unificado com a Espanha e Portugal (Cerqueira, 2006; Anjos, 2016). Esta decisão trouxe sérias consequências, como a dificuldade em usar a ampla literatura em Braille americano (Nemeth Code) (NBA, 1972) e a impossibilidade de usar os vários programas para transcrição computadorizada para matemática em Braille.

Ferronato (2002) afirma que os alunos precisam estar envolvidos com o processo de aprendizagem da matemática ou não terão sucesso na sua formação. Estudos de Barreto et al (2004) apresentam uma discussão sobre a criação de recursos (software) de apoio ao deficiente visual na busca de soluções matemáticas para as salas de aula. Estudos de Silveira (2011) apresentam o artefato tecnológico que elabora material instrucional com símbolos matemáticos, sendo estes convertidos automaticamente para o formato texto, que pode ser reproduzido pelos leitores de tela. Além disso, essa ferramenta gera o mesmo conteúdo no formato MathML que pode ser visualizado em navegadores de Internet na forma simbólica pelos estudantes sem deficiência visual. No entanto, o estudo apresenta algumas limitações como a navegação em fórmulas extensas e a incorporação desses componentes em um ambiente mais versáteis para editoração do que os navegadores Web.

Para melhorar os aspectos de ensino, existem outras estratégias como o MatVox (Silveira, 2010), que se afastam da alternativa de escrita em Braille, agregando facilidades de cálculo matemático a um editor de textos. Porém, o problema de leitura de matemática convencional, não foi resolvido por esta abordagem. Outra estratégia, que seria a utilização ampla do LaTeX pelos alunos com deficiência visual, pressupõe o uso corrente de um formato de texto que, pela abundancia de símbolos e "tags" extras, é muito difícil de ser lido através de um sintetizador de voz convencional. 
VII Congresso Brasileiro de Informática na Educação (CBIE 2018)

Anais do XXIX Simpósio Brasileiro de Informática na Educação (SBIE 2018)

\section{Os prós e contras da transcrição automatizada para Braille}

Segundo Souza (2017), a técnica Braille, ao desviar-se do grafismo, para adotar o pontismo como forma de representação tátil do alfabeto convencional, instituiu uma nova forma de tradução intersemiótica. Nas épocas anteriores ao invento do código Braille, eram bastante limitadas as possibilidades de corporificação da matemática para serem decodificados pelos indivíduos cegos. Com o advento do escrita Braille, no entanto, os cegos se tornaram proprietários de um competente sistema simbólico manejado por eles próprios, e encontraram no Braille a ferramenta fundamental que lhes proporcionou uuma revolução semiótica que aumentou significativamente a gama dos fenômenos, corpos e objetos que puderam então ser corporificados para serem compartilhados com as pessoas que enxergam. (Souza, 2017) Hoje em dia, essa especificidade é bem percebida na transcrição de livros didáticos para Braille, especialmente daqueles livros que fazem amplo uso de técnicas de diagramação do texto para melhor explicar o conteúdo, exigindo a presença de um "professor adaptador" para que a informação não seja distorcida no processo. (Borges, 2009)

A transcrição para Braille de materiais de Matemática, Química, Música etc, não é uma atividade simples, pois muitas das convenções usadas são peculiares para se tornarem compatíveis com as características da escrita Braille. Isso se tornou ainda mais difícil no Brasil, com a assinatura de um protocolo de colaboração para Unificação da Grafia Braille Unificada com Portugal em 2000 (MEC, 2006), que trouxe como consequência a mudança dos sinais gráficos e a adoção das regras de representação matemática adotadas na Espanha. Poucos avanços foram conseguidos, como o desenvolvimento do sistema automatizado de desenho por computador, que produz seu resultado em tinta ou em impressoras Braille (em modo gráfico), chamado Desenvox. Apesar da pequena disseminação, devido à restrição de interagir com um equipamento pouco disponível no Brasil (Barbosa et al, 2008; Borges, 2001), ainda existem a necessidade de criação de novas ferramentas tecnológicas para apoiar a escrita Braille.

Diante deste contexto, duas soluções começam a ser adotadas em grandes universidades e colégios federais tradicionais. A codificação AsciiMath (Gray, 2007), é uma linguagem de marcação para matemática criada originalmente para exibição em navegadores de internet, alternativa ao uso do LaTeX. Já o pequeno compilador denominado SonoraMat toma expressões matemáticas e cria frases que são sonoramente não ambíguas ao serem faladas e fáceis de serem compreendidas (Fig. 1).

$$
\sqrt{\mathrm{x}^{2}+\mathrm{y}^{2}} \quad \begin{aligned}
& \text { "raiz quadrada de } x \text { ao quadrado mais } y \\
& \text { ao quadrado" }
\end{aligned}
$$

Fig. 1: Texto criado com Intermat que corresponde a escrita Braille e sons.

Essas duas facilidades foram agregadas ao Dosvox e a uma nova ferramenta interativa (InterMat), permitindo o compartilhamento de informações matemáticas entre alunos e professores, com deficiência ou não. O Intermat permite também que as fórmulas criadas possam ser traduzidas automaticamente para o Braille Unificado, e vice-versa. A partir dos resultados obtidos, já é possível identificar as principais funções que estão tornando possível a agregação do conhecimento computacional nas salas de aula nos níveis médio e universitário. As fórmulas escritas em AsciiMath permitem apresentar na 
VII Congresso Brasileiro de Informática na Educação (CBIE 2018)

Anais do XXIX Simpósio Brasileiro de Informática na Educação (SBIE 2018)

forma de escrita convencional, muito simples e linear; que pode ser digitada facilmente por cegos, onde traduz-se para a forma matemática usual e adotada no Dosvox.

\section{Conclusão e Próximos Passos}

Neste trabalho foi apresentada a problemática de transcrição de matemática para cegos e algumas soluções adotadas que podem ser considerados como uma evolução tecnológica que coloca o Brasil próximo aos principais países produtores de Tecnologia Assistiva. Resta agora realizar estudos sistemáticos que permitam avaliar a qualidade da solução e a eficácia de inclusão que foi prometida. É importante definir estratégias de disseminação, e comparar com soluções usadas em outros países.

Os programas mostrados precisam ser avaliados e atualizados incessantemente, para aumentar a quantidade de materiais que já são gerados por eles, e que hoje são suficientes para atender aos requisitos mínimos para inclusão acadêmica nos cursos que tem como base a matemática nas universidades brasileiras públicas e privadas.

\section{Agradecimentos:}

Às colegas Daniela Cardoso Tavares do NCE/UFRJ e Elizabeth Canejo da Faetec/RJ pela prestimosa revisão deste texto.

Centro de Referência de Tecnologia Assistiva. Projeto com a Chancela SBC.

\section{Referencias}

Anjos, D. Z. - Código matemático unificado: da definição às diferenças semióticas na conversão da tinta ao Braille - Encontro Nacional de Educação Matemática - Educação Matemática na Contemporaneidade: desafios e Possibilidades - 2016, disponível em http://www.sbem.com.br/enem2016/anais/pdf/5413_2991_ID.pdf

Barbosa, P. M., Borges, J. A., Jansen, L. R., Lyrio, S. B - DESENVOX - Computer tools to teach basic geometry and drawing for visually impaired students in Brazil - International Congress on Mathematical Education - Monterrey, Mexico - Jul/2008.

Borges, J.A. Do Braille ao DOSVOX - diferenças nas vidas dos cegos brasileiros - Rio de Janeiro: UFRJ/COPPE, 2009.

Borges, José Antonio e Chagas Jr, Geraldo José Ferreira. Impressão Braille no Brasil: o papel do Braivox, Braille Fácil e Pintor Braille. Anais do I Simpósio Brasileiro sobre Sistema Braille, 2001.

Dias, A. F. S.; Franca, J.B.S.; Borges, M. R. S. Silva. Tecnologia Assistiva: Um Survey com portadores de deficiência visual em ambiente virtual de aprendizagem a partir do Modelo TAM. In: XVIII Conferência Internacional sobre Informática na Educação, TISE 2013, Porto Alegre

ECS Partners, Ltd. - Guidelines for Reading Mathematics - 2015, disponivel em https://stemreader.org.uk/guidelines/

Ferreira, T.A.C.S - Sistema Online de Síntese de Fala em Português - Tese de Mestrado Faculdade de Ciências e Tecnologia do Departamento de Engenharia Eletrotécnica e de Computadores - Universidade de Coimbra - 2014

Ferronato, Rubens. A construção de instrumento de inclusão no ensino da matemática. Dissertação de mestrado, UFSC, Florianópolis-SC. 2002, disponível em 
VII Congresso Brasileiro de Informática na Educação (CBIE 2018)

Anais do XXIX Simpósio Brasileiro de Informática na Educação (SBIE 2018)

https://repositorio.ufsc.br/bitstream/handle/123456789/82939/PEPS2320-

D.pdf? sequence $=1$ \&isAllowed $=\mathrm{y}$.

Gray, James (2007), "ASCIIMathML: now everyone can type MathML", MSOR Connections, 7 (3): $26-30$.

MEC. Grafia Braille para a Língua Portuguesa. Aprovada pela portaria nº 2.678 de 24/09/2006. Acessado em 10/08/2018. Disponível em http://portal.mec.gov.br/images/revista inclusao/grafiaport.txt

NBA - The Nemeth Braille Code for Mathematics and Science Notation - 1972 revision National Braille Association - 1972 disponível em http://www.brailleauthority.org/mathscience/nemeth1972.pdf

Silveira, H.M.; Martini, L.C. MATVOX: um aplicativo para deficientes visuais que proporciona a implementação de algoritmos e cálculos matemáticos em um editor de texto. Brazilian Symposium on Computers in Education - Simpósio Brasileiro de Informática na Educação - SBIE, Campinas -SP. 2011.

Silva, A.R.S.; Façanha, A.R.; Viana W.; Aires, J.; Sanchez, J. Especificação e desenvolvimento de um ambiente educativo móvel para a prática da escrita Braille. III Congresso Brasileiro de Informática na Educação (CBIE 2014). XXV Simpósio Brasileiro de Informática na Educação (SBIE 2014).

Souza, J. B. - O que vê a cegueira - A escrita Braille e sua natureza semiótica. - Ed. UFPB 2017

União Brasileira de Cegos. Código Matemático Unificado. São Paulo: F. Dorina Nowill. para Cegos - Coordenação de Jonir Bechara Cerqueira - 1998. 